\title{
Clinical Study \\ Primary Malignant Tumours of Bone Following Previous Malignancy
}

\author{
J. T. Patton, S. M. M. Sommerville, and R. J. Grimer \\ The Royal Orthopaedic Hospital Oncology Service, Royal Orthopaedioc Hospital, Bristol Road South, \\ Northfield, Birmingham B31 2AP, UK
}

Correspondence should be addressed to R. J. Grimer, rob.grimer@roh.nhs.uk

Received 5 July 2007; Accepted 25 March 2008

Recommended by Jay Wunder

Destructive bone lesions occurring in patients who have previously had a malignancy are generally assumed to be a metastasis from that malignancy. We reviewed 60 patients with a previous history of malignancy, who presented with a solitary bone lesion that was subsequently found to be a new and different primary sarcoma of bone. These second malignancies occurred in three distinct groups of patients: (1) patients with original tumours well known to be associated with second malignancies (5\%); (2) patients whose second malignancies were likely to be due to the previous treatment of their primary malignancy (40\%); (3) patients in whom there was no clearly defined association between malignancies (55\%). The purpose of this study is to emphasise the necessity for caution in assuming the diagnosis of a metastasis when a solitary bone lesion is identified following a prior malignancy. Inappropriate biopsy and treatment of primary bone sarcomas compromises limb salvage surgery and can affect patient mortality.

Copyright (c) 2008 J. T. Patton et al. This is an open access article distributed under the Creative Commons Attribution License, which permits unrestricted use, distribution, and reproduction in any medium, provided the original work is properly cited.

\section{INTRODUCTION}

With the advances in the treatment of many cancers, patient survival has improved dramatically such that a second malignant neoplasm is now observed more frequently [1, $2]$. It is well known that certain cancers are associated with an increased risk of a second cancer [3, 4], and that some treatments for cancer are in themselves known to be aetiological agents in the pathogenesis of a second cancer $[5,6]$. It is commonly assumed that bone lesions occurring in patients who have already had a malignant neoplasm are metastases from that malignancy. If the lesions are multiple, this will almost always be true, but if the lesion is solitary then further investigation is necessary to exclude other pathologies such as another primary bone tumour.

\section{MATERIALS AND METHODS}

The patients for this study were identified from a prospectively recorded database. The records of 60 patients who presented to our institution between 1980 and 2001 with a primary bone tumour following a prior malignancy were reviewed. The data was analysed in three separate groups, although it should be pointed out that certain patients might belong to more than one group.

\section{Group 1}

Patients were categorised into this group if they had a primary malignant neoplasm that is known to have a strong relationship with the development of a secondary malignancy.

\section{Group 2}

This group included patients who had treatment of their primary tumour that is known to predispose to the development of secondary sarcoma. This includes all the radiationinduced sarcomas that arose within the radiation field.

\section{Group3}

Patients who developed a primary tumour of bone, subsequent to a different primary malignant neoplasm that was 
not clearly related to that neoplasm or to the treatment used for that neoplasm, were included in this group.

\section{RESULTS}

At latest follow up, 31 patients (52\%) within the study group had died of disease relating to the primary bone sarcoma. The average time to development of metastases was 17.6 months (range 0-122 months).

\section{Group 1}

This group consisted of three patients (5\%), all of whom had retinoblastoma as their first tumour. The sarcoma that subsequently developed was an osteosarcoma in two and a primary leiomyosarcoma of bone in the other. The ages at which the second tumour arose were ten, sixteen, and twenty three years, with a lag time from the original tumour of eight, fifteen, and twenty two years, respectively. All the second tumours were located in sites remote from the head and neck.

\section{Group 2}

There were twenty four patients (40\%) who developed a sarcoma following previous radiotherapy for a primary tumour. Of these, twenty two were osteosarcoma and two were high-grade sarcoma with no identifiable differentiation. All of these secondary tumours were located in the previous radiation field. The average age of the patients at time of diagnosis of the second tumour was 45 years (range 16-80 years) with a lag time from the initial tumour of 14 years (range 4-31 years).

\section{Group 3}

Thirty three patients (55\%) developed a primary malignant bone tumour following a previous malignancy that was not felt to be clearly related to or associated with the treatment of the first malignancy (Table 1). The average age at diagnosis of the second malignancy was 52 years (range 8-84 years) and the lag time between tumours was 5 years (range 0-18 years). The most common initial malignancy was carcinoma of the breast (thirteen patients), which was associated with chondrosarcoma in six cases. Three patients presenting with breast cancer developed bone sarcomas in the proximal limb girdle. Only one of these patients received radiotherapy as treatment for their breast cancer. The bone sarcoma in this patient occurred in the contralateral proximal humerus. Chondrosarcoma was the most common second malignancy, affecting twelve patients. The tumours occurred at a wide distribution of sites, most frequently in the proximal femur (eight patients). Three of the 33 patients presented with a soft tissue sarcoma as the initial malignancy. The subsequent primary bone tumours were located in sites remote from the initial sarcoma and were felt to have histology unrelated to the initial tumour.

\section{DISCUSSION}

Significant progress has been made in the treatment of and survival from numerous malignant neoplasms in recent years. The improvement in patient survival has revealed previously unrecognised sequelae. The second malignant neoplasm is a well-described entity $[1,2]$. It is becoming clearer that genetic factors play a major role in the aetiology of the second malignant neoplasm. These factors are already well established in patients with tumours such as retinoblastoma and Ewings sarcoma $[4,7]$. In this study, we have reported six cases of breast carcinoma associated with chondrosarcoma. Recently, this association has been reported by others and a genetic trait postulated [8]. It has also been observed that cancer patients themselves are at greater risk of developing a subsequent malignancy than the general population [1].

Treatments for cancer have also been implicated in the aetiology of a second malignant neoplasm. Radiotherapy is a well-established causative agent and is dose dependent [6]. More recently, certain chemotherapeutic agents have also been implicated [9].

There is a tendency to assume that a solitary bone lesion following a malignancy is a metastasis from that malignancy. This is especially true if the patient is over 40 [10], and the interval between the initial malignancy and the second presentation is short. It was unfortunately beyond the capability of this study to provide a denominator of the number of patients with metastatic solitary bone lesions from the same population that presented to our tertiary referral centre with second and different primary bone tumours.

It is sobering to see that for the Group 3, patients in this study the average interval between tumours was only five years, and the average age of the patients was 52 years. In patients who have had radiotherapy to a previous lesion (Group 2), the assumption that the subsequent bone lesion is recurrent disease from the first malignancy can result in incorrect or substandard therapy.

The potential deleterious effects of a poorly performed biopsy have been well described by Mankin et al. [11]. There are now several reports illustrating the disastrous effects of inappropriate treatment of primary bone sarcomas, usually brought about by the assumption that the lesion is a metastasis [12]. In this study, patients managed in our tertiary referral centre had a biopsy prior to initial treatment. However, we know of at least 3 cases where presumption of the diagnosis of metastasis led to substandard treatment. This was caused by the assumption that the lesion was a metastasis, and the failure by the general orthopaedic surgeon to maintain an index of suspicion that a solitary bone lesion could be a primary bone sarcoma. Whist no particular traits were established, lesions in atypical sites (e.g., scapula, fibula) should be regarded with extrasuspicion. Most of the lesions in this study had characteristic appearances of primary bone sarcomas rather than metastases.

Internal fixation of these lesions can spread tumour cells along the whole length of the bone, rendering limb 
TABLE 1: Data for the Group 3 patients, in whom there was no clearly defined association between malignancies.

\begin{tabular}{|c|c|c|c|c|c|c|}
\hline Patient & $\begin{array}{l}\text { Age (at diagnosis of } \\
\text { second tumour) yrs }\end{array}$ & Sex & $\begin{array}{l}\text { Second tumour (pri- } \\
\text { mary bone sarcoma) }\end{array}$ & $\begin{array}{l}\text { Initial malignancy (Ca: } \\
\text { carcinoma) }\end{array}$ & $\begin{array}{l}\text { Site of second } \\
\text { tumour }\end{array}$ & $\begin{array}{l}\text { Lag (time to second } \\
\text { tumour) yrs }\end{array}$ \\
\hline 1 & 72 & $\mathrm{~F}$ & Chondrosarcoma & Ca breast & Prox femur & 14 \\
\hline 2 & 59 & $\mathrm{~F}$ & Chondrosarcoma & Ca breast & Prox humerus & 1 \\
\hline 3 & 50 & $\mathrm{~F}$ & Chondrosarcoma & Ca breast & Prox femur & 16 \\
\hline 4 & 58 & $\mathrm{~F}$ & Chondrosarcoma & Ca breast & Prox humerus & 2 \\
\hline 5 & 60 & $\mathrm{~F}$ & Chondrosarcoma & Ca breast & Distal femur & 0 \\
\hline 6 & 60 & $\mathrm{~F}$ & Chondrosarcoma & Ca bronchus & Prox tibia & 2 \\
\hline 7 & 72 & $\mathrm{~F}$ & Chondrosarcoma & Ca colon & Distal femur & 4 \\
\hline 8 & 44 & M & Chondrosarcoma & Ca oesophagus & Pelvis & 6 \\
\hline 9 & 58 & M & Chondrosarcoma & Ca rectum & Prox tibia & 7 \\
\hline 10 & 82 & M & Chondrosarcoma & Leukaemia & Prox femur & 6 \\
\hline 11 & 52 & $\mathrm{~F}$ & Chondrosarcoma & Malignant melanoma & Pelvis & 3 \\
\hline 12 & 66 & $\mathrm{~F}$ & Chondrosarcoma & Ca breast & Metatarsal & 0 \\
\hline 13 & 66 & $\mathrm{~F}$ & Chordoma & Ca breast & Sacrum & 0 \\
\hline 14 & 55 & $\mathrm{~F}$ & Chordoma & Ca breast & Sacrum & 12 \\
\hline 15 & 29 & $\mathrm{~F}$ & Ewings sarcoma & Ca kidney & Prox femur & 0 \\
\hline 16 & 12 & $\mathrm{~F}$ & Ewings sarcoma & Hodgkin's lymphoma & Fibula & 6 \\
\hline 17 & 9 & M & Ewings sarcoma & Leukaemia & Prox tibia & 5 \\
\hline 18 & 78 & M & Fibrosarcoma & $\begin{array}{l}\text { Malignant fibrous histi- } \\
\text { ocytoma (soft tisssue) }\end{array}$ & Pelvis & 2 \\
\hline 19 & 41 & $\mathrm{~F}$ & Fibrosarcoma & Malignant melanoma & Prox femur & 3 \\
\hline 20 & 84 & $\mathrm{~F}$ & Leiomyosarcoma & Ca breast & Distal fibula & 8 \\
\hline 21 & 47 & $\mathrm{~F}$ & $\begin{array}{l}\text { Malignant fibrous } \\
\text { histiocytoma }\end{array}$ & Hodgkin's lymphoma & Distal femur & 6 \\
\hline 22 & 39 & M & $\begin{array}{l}\text { Malignant fibrous } \\
\text { histiocytoma }\end{array}$ & Seminoma & Distal femur & 10 \\
\hline 23 & 27 & $\mathrm{~F}$ & $\begin{array}{l}\text { Malignant fibrous } \\
\text { histiocytoma }\end{array}$ & Ca breast & Prox femur & 2 \\
\hline 24 & 66 & M & Osteosarcoma & Ca bladder & Scapula & 3 \\
\hline 25 & 63 & $\mathrm{~F}$ & Osteosarcoma & Ca breast & Prox femur & 5 \\
\hline 26 & 10 & M & Osteosarcoma & Hodgkin's lymphoma & Prox humerus & 3 \\
\hline 27 & 33 & $\mathrm{~F}$ & Osteosarcoma & Malignant melanoma & Prox tibia & 4 \\
\hline 28 & 8 & $\mathrm{~F}$ & Osteosarcoma & Rhabdomyosarcoma & Prox tibia & 5 \\
\hline 29 & 12 & M & Osteosarcoma & Rhabdomyosarcoma & Prox femur & 11 \\
\hline 30 & 81 & $\mathrm{~F}$ & Sarcoma & Ca breast & Prox fibula & 0 \\
\hline 31 & 72 & $\mathrm{~F}$ & Sarcoma & Ca breast & Scapula & 3 \\
\hline 32 & 69 & M & Spindle cell sarcoma & Ca prostate & Scapula & 1 \\
\hline 33 & 81 & $\mathrm{~F}$ & Spindle cell sarcoma & Ca colon & Prox tibia & 18 \\
\hline
\end{tabular}

salvage surgery impossible. Clearly, therefore, we would urge caution in the treatment of solitary bone lesions, even in the presence of previous malignancy. There is rarely error in temporarily delaying potentially deleterious surgery or radiotherapy, imaging the patient appropriately, and performing a biopsy by a surgeon experienced in orthopaedic oncological procedure [13].

\section{REFERENCES}

[1] C. Dong and K. Hemminki, "Second primary neoplasms among 53159 haematolymphoproliferative malignancy pa- tients in Sweden, 1958-1996: a search for common mechanisms," British Journal of Cancer, vol. 85, no. 7, pp. 997-1005, 2001.

[2] O. Merimsky, Y. Kollender, J. Issakov, et al., "Multiple primary malignancies in association with soft tissue sarcomas," Cancer, vol. 91, no. 7, pp. 1363-1371, 2001.

[3] J. Moppett, A. Oakhill, and A. W. Duncan, "Second malignancies in children: the usual suspects?" European Journal of Radiology, vol. 38, no. 3, pp. 235-248, 2001.

[4] C. E. Margo, L. E. Harman, and Z. D. Mulla, "Retinoblastoma," Cancer Control, vol. 5, no. 4, pp. 310-316, 1998.

[5] D. C. Rich, C. A. Corpron, M. B. Smith, C. T. Black, K. P. Lally, and R. J. Andrassy, "Second malignant neoplasms in children 
after treatment of soft tissue sarcoma," Journal of Pediatric Surgery, vol. 32, no. 2, pp. 369-372, 1997.

[6] J. F. Kuttesch Jr., L. H. Wexler, R. B. Marcus, et al., "Second malignancies after Ewing's sarcoma: radiation dosedependency of secondary sarcomas," Journal of Clinical Oncology, vol. 14, no. 10, pp. 2818-2825, 1996.

[7] A. Abudu, D. C. Mangham, G. M. Reynolds, et al., "Overexpression of p53 protein in primary Ewing's sarcoma of bone: relationship to tumour stage, response and prognosis," British Journal of Cancer, vol. 79, no. 7-8, pp. 1185-1189, 1999.

[8] A. E. Odink, C. J. van Asperen, J. P. Vandenbroucke, A. M. Cleton-Jansen, and P. C. W. Hogendoorn, "An association between cartilaginous tumours and breast cancer in the national pathology registration in The Netherlands points towards a possible genetic trait," Journal of Pathology, vol. 193, no. 2, pp. 190-192, 2001.

[9] A. J. Swerdlow, J. A. Barber, G. V. Hudson, et al., "Risk of second malignancy after Hodgkin's disease in a collaborative British cohort: the relation to age at treatment," Journal of Clinical Oncology, vol. 18, no. 3, pp. 498-509, 2000.

[10] B. T. Rougraff, J. S. Kneisl, and M. A. Simon, "Skeletal metastases of unknown origin. A prospective study of a diagnostic strategy," Journal of Bone and Joint Surgery A, vol. 75, no. 9, pp. 1276-1281, 1993.

[11] H. J. Mankin, T. A. Lange, and S. S. Spanier, "The hazards of biopsy in patients with malignant primary bone and softtissue tumors," Journal of Bone and Joint Surgery A, vol. 64, no. 8, pp. 1121-1127, 1982.

[12] G. M. Spence, M. T. Dunning, S. R. Cannon, and T. W. R. Briggs, "The hazard of retrograde nailing in pathological fractures: three cases involving primary musculoskeletal malignancy," Injury, vol. 33, no. 6, pp. 533-538, 2002.

[13] M. A. Simon and J. S. Biermann, "Biopsy of bone and softtissue lesions," Instructional Course Lectures, vol. 43, pp. 521526, 1994. 


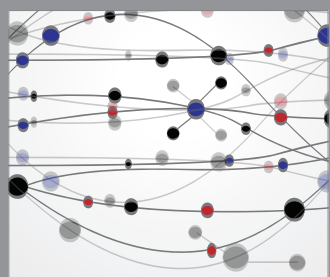

The Scientific World Journal
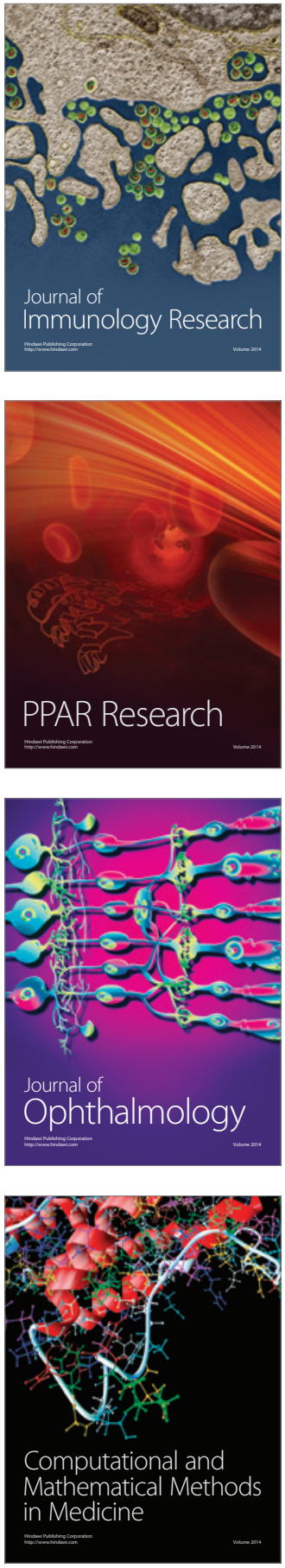

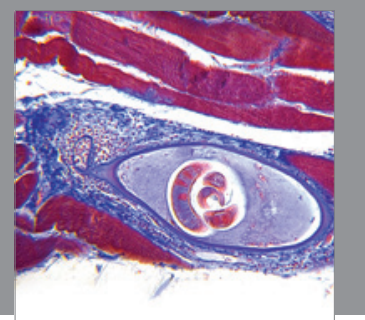

Gastroenterology

Research and Practice
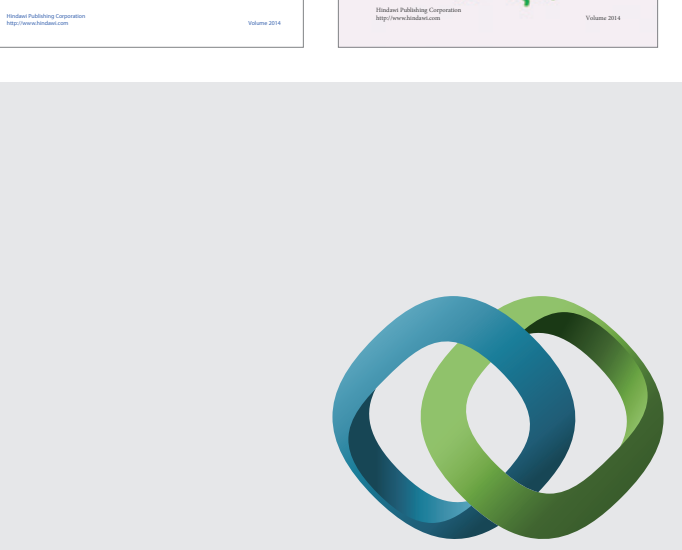

\section{Hindawi}

Submit your manuscripts at

http://www.hindawi.com
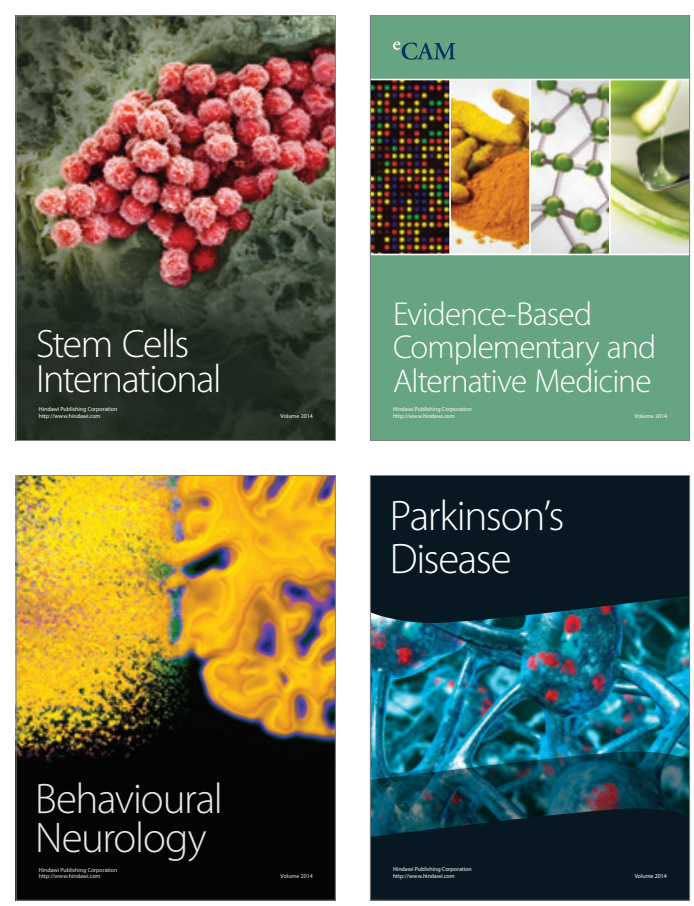

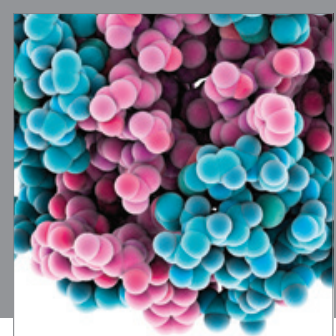

Journal of
Diabetes Research

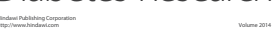

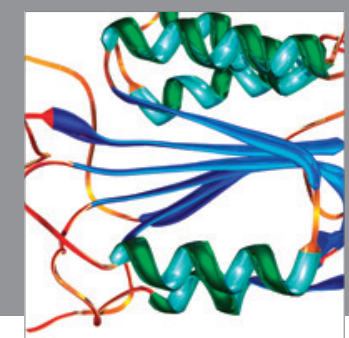

Disease Markers
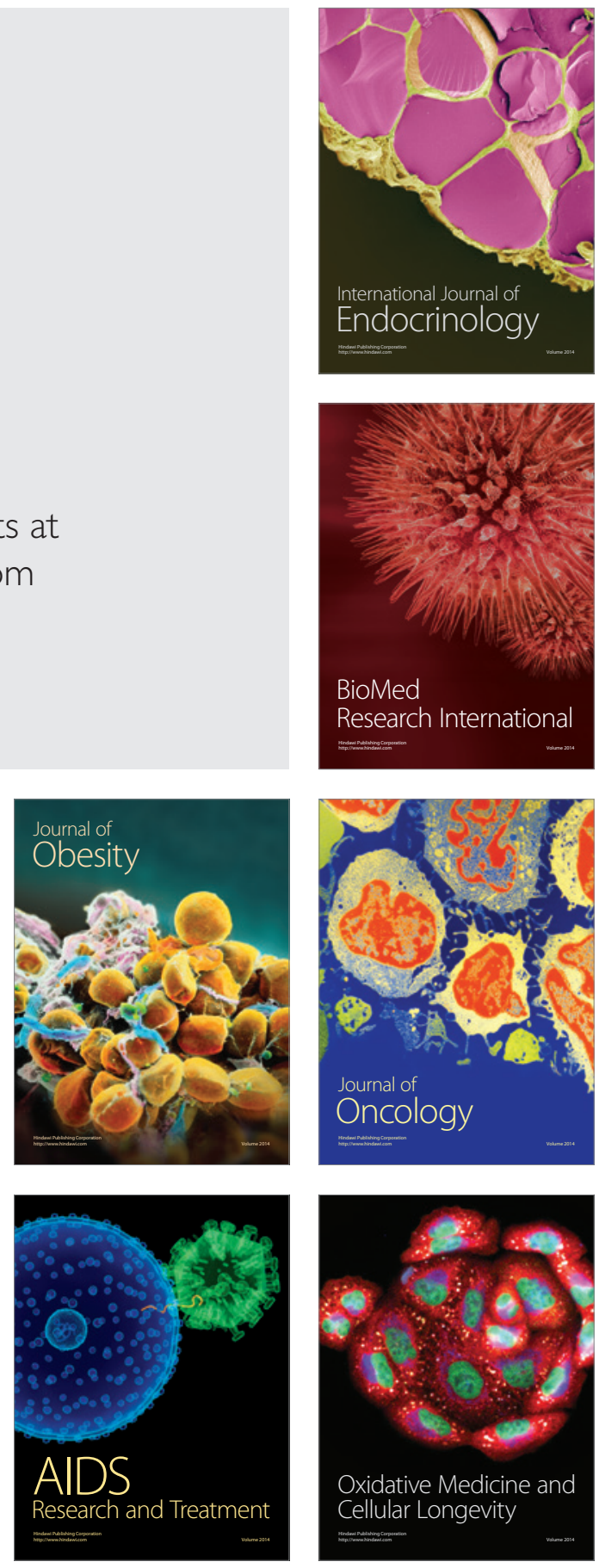